\title{
Cartan-Hannay-Berry Phases and Symmetry
}

\author{
J. Marsden*, R. Montgomery**, and T. Ratiu*** \\ Dedicated to Roger Brockett on the occasion of his 50 th birthday
}

\begin{abstract}
We give a systematic treatment of the treatment of the classical Hannay-Berry phases for mechanical systems in terms of the holonomy of naturally constructed connections on bundles associated to the system. We make the costructions using symmetry and reduction and, for moving systems, we use the Cartan connection. These ideas are woven with the idea of Montgomery [1988] on the averaging of connections to produce the Hannay-Berry connection.
\end{abstract}

\section{$\$ 1$ Introduction}

In this paper we give some of the results of the work of Montgomery [1988], and of Marsden, Montgomery, and Ratiu [1989] on the use of symmetry and reduction in the theory of Hannay-Berry phases for mechanical systems. We have in mind both classical and quantum systems, but this paper will only be concerned with classical mechanical systems. We intend this paper to be a short version of the cited works which should help interested readers get the ideas quickly. For the most part proofs will be omitted. Readers well versed in connection theory will be able to supply proofs themselves; for details, the longer version can be consulted.

The work is motivated by that of Hannay and Berry on phases for parametrized families of integrable systems. There are, however, many systems to which the Hannay-Berry construction

\footnotetext{
${ }^{*}$ Research partially supported by NSF grant DMS 8702502, DOE Contract DE-AT03-88ER-12097 and MSI at Cornell University.

${ }^{* * *}$ Research partially supported by an NSF postdoctoral fellowship and NSF grant DMS 8702502.

${ }^{* * *}$ Research partially supported by NSF grant DMS 8701318-01.

AMS Subject Classification 58F, 70H.
} 
does not literally apply, for examplè, the motion of a bead on a slowly rotating hoop. (Hannay and Berry get around this by regarding the problem as the limit of a sequence of families of integrable systems, namely the limit of infinite potential constraining forces, but we are able to treat it in a simple, direct way). This paper shows how to put these and other systems consistently into the framework of connections on bundles and reduction of Hamiltonian systems with symmetry. What we do is take the parameter space $M$ to be a space of motions of an ambient space $\left(\mathbb{R}^{2}\right.$ for the hoop) and the phase space $\mathrm{P}$ to be the cotangent bundle of the constrained configuration space. Physically, the key observation, due to Jeeva Anandan, is that when comparing two different constrained systems one must compare points with the same momentum relative to a fixed inertial frame.

Mathematically, there are two key important observations in the present paper. The first is that connections can be averaged; in Montgomery [1988] the context was that of a fixed connection relative to a varying group action. In this paper, we average a connection varying with respect to a fixed ( $\mathrm{M}$ independent) group action. The second key observation is that the correct connection to average is one due to Cartan [1923]. The Cartan connection encodes the ficticious forces-the centrifugal, Euler, and Coriolis forces--due to an accelerating inertial frame, namely the one attached to the moving constrained system. Putting these two observations together we find that the correct connection encoding the phase shift of Hannay and Berry for constrained systems is obtained by averaging the Cartan connection. This connection we call the Cartan-HannayBerry connection.

\section{$\$ 2$ Moving Systems}

Consider a Riemannian manifold $S$, a submanifold $Q$, a space $M$ of embeddings of $Q$ into $S$, and let $\mathrm{m}_{\mathrm{t}} \in \mathrm{M}$ be a given curve. If a particle in $\mathrm{Q}$ is following a curve $\mathrm{q}(\mathrm{t})$, and if $\mathrm{Q}$ moves by superposing the motion $m_{t}$, then the path of the particle in $S$ is given by $m_{t}(q(t))$. Thus its velocity in $S$ is given by

$$
\mathrm{T}_{\mathrm{q}(\mathrm{t})} \mathrm{m}_{\mathrm{t}} \cdot \dot{\mathrm{q}}(\mathrm{t})+Z_{\mathrm{t}}\left(\mathrm{m}_{\mathrm{t}}(\mathrm{q}(\mathrm{t}))\right)
$$

where $Z_{t}\left(m_{t}(q)\right)=\frac{d}{d t} m_{t}(q)$. Consider the Lagrangian on TQ of the form

$$
\mathrm{L}_{\mathrm{m}_{\mathrm{t}}}(\mathrm{q}, \mathrm{v})=\frac{1}{2}\left\|\mathrm{~T}_{\mathrm{q}(\mathrm{t})} \mathrm{m}_{\mathrm{t}} \cdot \mathrm{v}+Z_{\mathrm{t}}\left(\mathrm{m}_{\mathrm{t}}(\mathrm{q})\right)^{\mathrm{T}}\right\|^{2}-\mathrm{V}(\mathrm{q})-\mathrm{U}\left(\mathrm{m}_{\mathrm{t}}(\mathrm{q})\right)
$$

where $\mathrm{V}$ is a given potential on $\mathrm{Q}, \mathrm{U}$ is a given potential on $S$, and ${ }^{\mathrm{T}}$ denotes projection onto the tangent space to the (moving) image of Q. Taking the Legendre transform of (2), we get a Hamiltonian with momentum

$$
\mathrm{p}=\left(\mathrm{T}_{\mathrm{q}(\mathrm{t})} \mathrm{m}_{\mathrm{t}} \cdot \mathrm{v}+Z_{\mathrm{t}}\left(\mathrm{m}_{\mathrm{t}}(\mathrm{q})\right)^{\mathrm{T}}\right)^{\mathbf{6}}
$$

where ${ }^{b}$ denotes the index lowering operation determined by the metric on $\mathcal{S}$. Physically, if $S$ is $\mathbb{R}^{3}$, then $\mathrm{p}$ is the inertial momentum (see the hoop example in $\S 3$ ). This extra term $Z_{\mathrm{t}}\left(\mathrm{m}_{\mathrm{l}}(\mathrm{q})\right)^{\mathrm{T}}$ 
leads to a connection called the Cartan connection on the bundle $\mathrm{Q} \times \mathrm{M} \rightarrow \mathrm{M}$, with horizontal lift defined to be $Z(\mathrm{~m}) \mapsto\left(\mathrm{Tm}^{-1} Z(\mathrm{~m})^{\mathrm{T}}, Z(\mathrm{~m})\right)$. (See for example, Marsden and Hughes [1983] for an account of some aspects of Cartan's contributions.) The Hamiltonian picks up a cross term and so takes the form

$$
\mathrm{H}_{Z_{\mathrm{t}}}(\mathrm{q}, \mathrm{p})=\frac{1}{2}\|\mathrm{p}\|^{2}-\mathcal{P}\left(Z_{\mathrm{t}}\right)+\frac{1}{2}\left\|Z_{\mathrm{t}}^{\mathrm{T}}\right\|^{2}+\mathrm{V}(\mathrm{q})+\mathrm{U}\left(\mathrm{m}_{\mathrm{t}}(\mathrm{q})\right)
$$

where the cross term is $\mathcal{P}\left(Z_{\mathrm{t}}\right)(\mathrm{q}, \mathrm{p})=\left\langle\mathrm{p}, Z_{\mathrm{t}}^{\mathrm{T}}(\mathrm{q})\right\rangle$. The Hamiltonian vector field of this cross term $X_{\mathcal{T}\left(Z_{t}\right)}$ represents the noninertial forces and also has the natural interpretation as a horizontal lift of the vector field $Z_{\mathrm{t}}$ relative to a certain connection on the bundle $\mathrm{T}^{*} \mathrm{Q} \times \mathrm{M} \rightarrow \mathrm{M}$, which we also call the Cartan connection.

Let $G$ be a Lie group which acts on $T^{*} Q$ in a Hamiltonian fashion and leaves $\mathrm{H}_{0}$ (defined by setting $Z=0$ ) in (4)) invariant. In our examples $G$ is either $\mathbb{R}$ acting by the flow of $\mathrm{H}_{0}$ (the hoop), or a subgroup of the isometry group of $\mathrm{Q}$ which leaves $\mathrm{V}$ and $\mathrm{U}$ invariant, and acts on $T^{*} Q$ by cotangent lift (the pendulum). In any case, we assume $G$ has an invariant measure relative to which we can average.

Assuming the "averaging principle" (cf. Arnold [1978], for example) we replace $\mathrm{H}_{\mathcal{Z}_{\mathrm{t}}}$ by its G-average,

$$
\left\langle\mathrm{H}_{Z_{\mathrm{t}}}\right\rangle(\mathrm{q}, \mathrm{p})=\frac{1}{2}\|\mathrm{p}\|^{2}-\left\langle\mathcal{P}\left(Z_{\mathrm{t}}\right)\right\rangle+\frac{1}{2}\left\langle\left\|Z_{\mathrm{t}}^{\mathrm{T}}\right\|^{2}\right\rangle+\mathrm{V}(\mathrm{q})+\left\langle\mathrm{U}\left(\mathrm{m}_{\mathrm{t}}(\mathrm{q})\right)\right\rangle
$$

In (5) we shall assume the term $\frac{1}{2}\left\langle\left\|Z_{\mathrm{t}}^{\mathrm{T}}\right\|^{2}\right\rangle$ is small and discard it. Thus, consider

$$
\mathscr{H}(\mathrm{q}, \mathrm{p}, \mathrm{t})=\frac{1}{2}\|\mathrm{p}\|^{2}-\left\langle\mathcal{P}\left(Z_{\mathrm{t}}\right)\right\rangle+\mathrm{V}(\mathrm{q})+\left\langle\mathrm{U}\left(\mathrm{m}_{\mathrm{t}}(\mathrm{q})\right)\right\rangle=\mathcal{H}_{0}(\mathrm{q}, \mathrm{p})-\left\langle\mathcal{P}\left(Z_{\mathrm{t}}\right)\right\rangle+\left\langle\mathrm{U}\left(\mathrm{m}_{\mathrm{t}}(\mathrm{q})\right)\right\rangle
$$

where $\mathcal{H}_{0}=\frac{1}{2}\|\mathrm{p}\|^{2}+\mathrm{V}(\mathrm{q})$. We shall consider the dynamics on $\mathrm{T}^{*} \mathrm{Q} \times \mathrm{M}$ given by the vector field

$$
\left(\mathrm{X}_{\mathscr{F}}, Z_{\mathrm{t}}\right)=\left(\mathrm{X}_{\mathscr{H} \mathscr{G}_{0}}-\mathrm{X}_{\left\langle\mathcal{P}\left(Z_{\mathrm{t}}\right)\right\rangle}+\mathrm{X}_{\left.\left\langle\mathrm{U} \circ \mathrm{m}_{\mathrm{t}}\right)\right\rangle}, Z_{\mathrm{t}}\right)
$$

The vector field representing the extra terms in this representation due to the superposed motion of the system, namely

$$
\operatorname{hor}\left(Z_{\mathrm{t}}\right)=\left(-X_{\left\langle\mathcal{P}\left(Z_{\mathrm{t}}\right)\right\rangle}, Z_{\mathrm{t}}\right)
$$

has a natural interpretation as the horizontal lift of $Z_{\mathrm{t}}$ relative to a connection on $\mathrm{T}^{*} \mathrm{Q} \times \mathrm{M}$, which is obtained by averaging the Cartan connection and so we call it the Cartan-Hannay-Berry (CHB) connection. The holonomy of this connection is the Hannay-Berry phase of a slowly moving constrained system. 


\section{$\$ 3$ The ball in the hoop and the Foucault pendulum}

We now give two examples of the formalism. The procedures used in the ball in the hoop example are due to J. Anandan.

Example 1 Ball in the Hoop Consider a hoop (not necessarily circular) in which a bead slides without friction, as in Figure 1 below. As the bead is sliding, the hoop is rotated in its plane through an angle $\theta(t)$ with angular velocity $\omega(t)=\dot{\theta}(t) k$. Let $s$ denote the arc length along the hoop, measured from a reference point on the hoop and let $q(s)$ be the vector from the origin to the corresponding point on the hoop; thus the shape of the hoop is determined by this function $\mathbf{q}(s)$. The unit tangent vector is $\mathbf{q}^{\prime}(s)$ and the position of the reference point $\mathbf{q}(s(t))$ relative to an inertial frame in space is $R_{\theta(t)} q(s(t))$, where $R_{\theta}$ is the rotation in the plane of the hoop through an angle $\theta$.
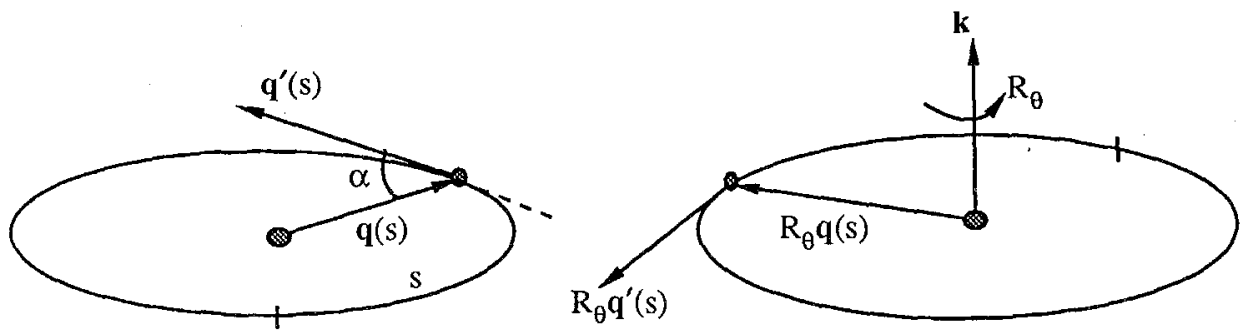

Figure 1 The ball in the hoop

The configuration space is diffeomorphic to the circle $Q=S^{1}$ with length $L$ the length of the hoop. The Lagrangian $L(s, \dot{s}, t)$ is simply the kinetic energy of the particle; i.e., since

$$
\frac{d}{d t} R_{\theta(t)} q(s(t))=R_{\theta(t)} q^{\prime}(s(t)) \dot{s}(t)+R_{\theta(t)}[\omega(t) \times q(s(t))],
$$

we set

$$
\mathrm{L}(\mathrm{s}, \dot{\mathrm{s}}, \mathrm{t})=\frac{1}{2} \mathrm{~m}\left\|\mathbf{q}^{\prime}(\mathrm{s}) \dot{\mathrm{s}}+\omega \times \mathbf{q}(\mathrm{s})\right\|^{2} .
$$

The Legendre transformation yields

$$
\mathrm{p}=\mathrm{m} \dot{\mathrm{s}}+\mathrm{q}^{\prime}(\mathrm{s}) \cdot[\omega(\mathrm{t}) \times \mathrm{q}(\mathrm{s})]=\text { inertial momentum }
$$

and

$$
\mathrm{H}=\frac{1}{2 \mathrm{~m}} \mathrm{p}^{2}-P+\frac{1}{2}\left\|(\omega \times \mathrm{q})^{\mathrm{T}}\right\|^{2}
$$


Here ${ }^{\prime}=\frac{d}{d s},{ }^{T}$ denotes projection onto the hoop's tangent, and

$$
\mathscr{P}=\mathrm{pq}^{\prime}(\mathrm{s}) \cdot(\omega(\mathrm{t}) \times \mathbf{q}(\mathrm{s}))=\mathrm{p}\|\mathbf{q}\| \sin \alpha(\mathrm{s})
$$

(see Figure 1). $\mathcal{P}$ ds is the instantaneous slippage of the bead due to the hoop's rotation.

For $\omega$ small relative to $\mathrm{p}$ it is a good approximation to replace $\mathrm{H}$ by

$$
\begin{gathered}
\mathcal{H}=\frac{1}{2 \mathrm{~m}} \mathrm{p}^{2}-\langle P\rangle . \\
\langle P\rangle=\frac{1}{\mathrm{~L}} \oint \mathcal{P}(\mathrm{s}) \mathrm{ds}=\frac{2 \mathrm{~A}}{\mathrm{~L}} \mathrm{p} \omega
\end{gathered}
$$

where $A$ is the hoop's area and $L$ its length. (This is seen by Stokes' theorem: $d(r \sin \alpha d s)=$ $r \cos \alpha d \alpha \wedge d s=2 d A$.) Now

$$
X_{\mathscr{H}}=\frac{1}{\mathrm{~m}} \mathrm{p} \frac{\partial}{\partial \mathrm{q}}-\omega \frac{2 \mathrm{~A}}{\mathrm{~L}} \frac{\partial}{\partial \mathrm{q}}
$$

is the (approximate) dynamic vector field. The second term in (5) gives the Hannay-Berry angle. After one full revolution in the space of motions $\left(\int \omega \mathrm{dt}=2 \pi\right)$ the integral of this second term is

$$
-\frac{4 \pi \mathrm{A}}{\mathrm{L}}=\text { Hannay-Berry angle for hoop. }
$$

Equation (6) gives the holonomy of the CHB connection, and expresses the displacement of a bead of inertial momentum $\mathcal{P}$ after one full revolution of the hoop, compared to where it would have been after this same amount of time with the same inertial momentum, but without rotating the hoop.

Example 2 The Foucault Pendulum The Foucault pendulum is a spherical pendulum at co-latitude $\alpha$ on the surface of the Earth. Denote by $\mathbf{q}$ the position of the pendulum on the sphere of radius $\ell$, the length of the pendulum arm (see Figure 2).

Let $\mathbf{r}_{0}$ denote the vector from the center of the Earth to the point of suspension of the pendulum. Due to the rotation of the Earth the point $q$ moves to the point $R_{t}\left(r_{0}+q\right)$, where $R_{t}$ is the rotation about the Oz-axis. Let $\omega=\frac{T}{2 \pi}$ denote the angular velocity of the Earth's rotation. The potential energy of the pendulum is $V(\mathbf{q})=\operatorname{mgl} \mathbf{q} \cdot \hat{\mathbf{r}}_{0}$, where $\hat{\mathbf{r}}_{0}=\frac{\mathbf{r}_{0}}{\left\|\mathbf{r}_{0} U\right\|}$. The velocity of the point $\mathbf{q}$ on the sphere during Earth's rotation is therefore

$$
\dot{q}+R_{t}\left[\omega \times\left(\hat{r}_{0}+q\right)\right]
$$

where we identify $\omega$ with the vector $\omega \mathbf{k}$. 


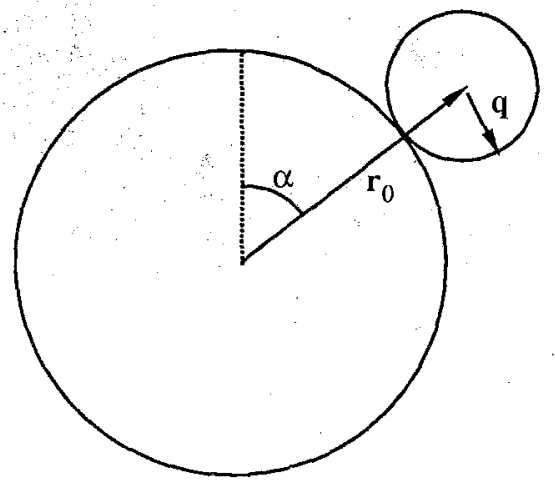

Figure 2

The Lagrangian is

$$
L=\frac{1}{2} m\left\|\omega \times\left(\hat{\mathbf{r}}_{0}+\mathbf{q}\right)+\dot{\mathbf{q}}\right\|^{2}-V(\mathbf{q})
$$

so that the Legendre transformation gives

$$
\mathbf{p}=\mathrm{m}\left(\dot{\mathbf{q}}+\omega \times\left(\hat{\mathbf{r}}_{0}+\mathbf{q}\right)\right)^{\mathbf{T}}=\mathrm{m}\left[\dot{\mathbf{q}}+\left(\omega \times\left(\hat{\mathbf{r}}_{0}+\mathbf{q}\right)\right)^{\mathrm{T}}\right]
$$

by identifying $T^{*} S^{2}$ with $\mathrm{TS}^{2}$ via the standard metric; here $\left(\omega \times\left(\hat{\mathbf{r}}_{0}+q\right)\right)^{\mathrm{T}}$ denotes the tangential component of $\omega \times\left(\hat{\mathbf{r}}_{0}+\mathbf{q}\right)$. Then

$$
\dot{\mathbf{q}}=\frac{1}{\mathrm{~m}} \mathbf{p}-\left[\omega \times\left(\hat{\mathrm{r}}_{0}+\mathbf{q}\right)\right]^{\mathrm{T}}
$$

and

$$
\dot{q}+\omega \times\left(\hat{\mathbf{r}}_{0}+q\right)=\frac{1}{\mathrm{~m}} \mathbf{p}+\left[\omega \times\left(\hat{\mathbf{r}}_{0}+\mathbf{q}\right)\right]^{\perp},
$$

where $\left(\omega \times\left(\hat{\mathbf{r}}_{0}+q\right)\right)^{\perp}$ denotes the normal component of $\omega \times\left(\hat{\mathbf{r}}_{0}+q\right)$ to the sphere. Therefore the Hamiltonian is given by

$$
\begin{aligned}
H=\mathbf{p} \dot{q}-L= & \frac{1}{m}\|\mathbf{p}\|^{2}-\frac{1}{m} \mathbf{p} \cdot\left(\omega \times\left(\hat{\mathbf{r}}_{0}+\mathbf{q}\right)\right)^{\mathrm{T}} \\
& \quad-\left(\frac{1}{2 \mathrm{~m}}\|\mathbf{p}\|^{2}+\frac{\mathrm{m}}{2}\left\|\left(\omega \times\left(\hat{\mathbf{r}}_{0}+\mathbf{q}\right)\right)^{\perp}\right\|^{2}-\mathrm{V}(\mathbf{q})\right) \\
= & \frac{1}{2 \mathrm{~m}}\|\mathbf{p}\|^{2}+\mathrm{V}(\mathbf{q})-\mathcal{P}-\frac{\mathrm{m}}{2}\left\|\left(\omega \times\left(\hat{\mathbf{r}}_{0}+\mathbf{q}\right)\right)^{\perp}\right\|^{2}
\end{aligned}
$$

where

$$
\mathscr{P}=\frac{1}{\mathrm{~m}} \mathbf{p} \cdot\left(\omega \times\left(\mathbf{r}_{0}+\mathbf{q}\right)\right)^{\mathrm{T}}=\frac{1}{\mathrm{~m}} \mathbf{p} \cdot\left(\omega \times\left(\mathbf{r}_{0}+\mathbf{q}\right)\right)
$$


since $\mathbf{p}$ is tangential.

Letting $M$ be the space $S^{1}$ of embeddings of the sphere of radius $\ell$ in $\mathbb{R}^{3}$ obtained by rotating a fixed initial sphere tangent to the Earth at co-latitude $\alpha$, as in Figure 2, we recognize that $\mathscr{P}$ is the Hamiltonian defining the induced Cartan connection on $T^{*} S^{2} \times S^{1}$ (see $\S 4$ ).

Since $(\omega \times \mathbf{q})^{\perp}=0$, we have $\left[\omega \times\left(\mathbf{r}_{0} \times \boldsymbol{q}\right)\right]^{\perp}=\left(\omega \times \mathbf{r}_{0}\right)^{\perp}=$ constant. In the equations of motion one can drop this constant. Had we considered a Foucault pendulum on an ellipsoid, this term would not be constant, but it would be of order $\omega^{2}$, which is the general case. According to the averaging principle, one ignores this term.

Let $\mathrm{S}_{\mathrm{r}_{0}}^{1}$ be another circle, acting on the phase space of the pendulum by rotation about the $\mathbf{r}_{0}$ axis. As in the ball in the hoop, and consistent with the theory sketched in $\$ 4$, the horizontal lift of the induced Hannay-Berry connection is given by the Hamiltonian vector field of $\mathcal{P}$, i.e., by $\left\langle p \cdot\left[\omega \times\left(\mathbf{r}_{0} \times \mathbf{q}\right)\right]\right\rangle$. But if $\mathbf{v}$ is any constant vector,

$$
\langle\mathbf{p} \cdot \mathbf{v}\rangle=\left\langle\mathbf{p} \cdot \hat{\mathbf{r}}_{0}\right\rangle\left(\mathbf{v} \cdot \hat{\mathbf{r}}_{0}\right)
$$

since the $S_{\mathbf{r}_{0}}^{1}$-action over which we average has $\mathbf{r}_{0}$ as its axis of rotation. Setting $\mathbf{v}=\omega \times \mathbf{r}_{0}$, this implies that

and hence

$$
\left\langle\mathbf{p} \cdot\left(\omega \times \mathbf{r}_{0}\right)\right\rangle=0
$$

$$
\left\langle\mathbf{p} \cdot\left[\omega \times\left(\mathbf{r}_{0}+\mathbf{q}\right)\right]\right\rangle=\langle\mathbf{p} \cdot(\omega \times \mathbf{q})\rangle .
$$

Let $\mathrm{I}=\mathbf{p} \cdot\left(\mathbf{r}_{0} \times \mathbf{q}\right)$ be the momentum map of the $\mathrm{S}_{\mathrm{T}_{0}}^{1}$-action. By (14)

$$
\langle\mathbf{p} \cdot(\omega \times \mathbf{q})\rangle=\langle\omega \cdot(\mathbf{q} \times \mathbf{p})\rangle=\left[(\mathbf{q} \times \mathbf{p}) \cdot \mathbf{r}_{0}\right]\left\langle\omega \cdot \hat{\mathbf{r}}_{0}\right\rangle=\mathrm{I} \omega \cos \alpha
$$

since $\omega \cdot \hat{\mathbf{r}}_{0}$ is constant. Thus,

$$
\langle P\rangle=I \omega \cos \alpha
$$

and so the horizontal lift of $\omega$ is given by $\left(-X_{\langle p\rangle}, \omega\right)=\left(-\omega \cos \alpha \frac{\partial}{\partial \theta}, \omega\right)$, where $\frac{\partial}{\partial \theta}$ is the infinitesimal generator of the $S_{I_{0}}^{1}$-action, corresponding to $I$. Therefore the horizontal lift of the curve $\varphi(t)=\omega t$ in $M$ is determined by the differential equation

$$
\dot{\theta}=-\omega \cos \alpha
$$

so that if this curve is a loop parametrized on $\left[0, \frac{T}{2 \pi}\right]$ we get 


$$
\theta(T)-\theta(0)=-\int_{0}^{T} \omega \cos \alpha d t=-\omega T \cos \alpha=-2 \pi \cos \alpha
$$

which is the deviation of the plane of oscillation in the laboratory frame (i.e. a frame fixed on the Earth) of the Foucault pendulum during 24 hours. Equation (16) is essentially Foucault's classic formula.

For example, if we are at the equator, where $\alpha=\frac{\pi}{2}$, there is no deviation. If we are at the North Pole, where $\alpha=0$, the plane rotates in the opposite direction to that of the Earth's rotation, performing a full circular motion. These results are in an inertial frame. The usual Foucault result is for a lab frame attached to the Earth. The two frames are related by $\mathbf{q}_{\text {inertial }}=R_{t}\left(r_{0}+q_{\text {lab }}\right)$. In 24 hours this means we must add $2 \pi$ to our $\Delta \theta$ to get the angle shift in the lab frame.

For a nice elementary discussion of Foucault's pendulum also see Berry [1988].

\section{$\$ 4$ Construction and Properties of the Hannay-Berry Connection}

In this section we summarize the properties and construction of a general Hannay-Berry connection and specialize this to the case of moving systems. The general theory is a generalization of that developed for trivial bundles with symplectic fiber by Golin, Knauf, and Marmi [1989] and Montgomery [1988]. The freedom we allow of choosing an arbitrary background connection is essential in the construction of the CHB-connection discussed below. These background connections need not be principal connections but they are Ehresmann connections.

\section{\$4A Ehresmann Connections and Holonomy}

If $\pi: \mathrm{E} \rightarrow \mathrm{M}$ is a locally trivial fiber bundle, an Ehresmann connection is a smooth subbundle $\mathrm{H}$ of $\mathrm{TE}$ such that $\mathrm{H} \oplus \mathrm{V}=\mathrm{TE}$, where $\mathrm{V}=\operatorname{ker} \mathrm{T} \pi$ is called the vertical subbundle and $\mathrm{H}$ is the horizontal subbundle of the connection. Alternatively, an Ehresmann connection is given by a one-form $\Gamma \in \Omega^{1}(E, V)$ on $E$ with values in $V$ and which is the identity on $\mathrm{V}$, or by a horizontal lift, i.e., a map hor sending smooth vector fields on $M$ to smooth sections of $\mathrm{H}$.

Let $m(t), t \in[0,1]$ be a smooth path in $M$. A horizontal lift of $m(t)$ is a smooth path $p(t)$ in E such that $\pi(p(t))=m(t)$ and the tangent vector $\dot{p}(t)$ to $p(t)$ is horizontal for every $t \in[0,1]$. If $\pi: F \rightarrow M$ is a locally trivial fiber bundle, then given a smooth path $m(t)$, in $M$ with $\mathrm{m}_{0}=\mathrm{m}(0), 0 \leq \mathrm{t} \leq 1$ and $\mathrm{p} \in \pi^{-1}\left(\mathrm{~m}_{0}\right)$, there is a unique horizontal lift $\mathrm{p}(\mathrm{t})$ of $\mathrm{m}(\mathrm{t})$ satisfying $p(0)=p$. The map sending $p$ to $p(1)$ defines, by uniqueness of horizontal lift, a bijection from $\pi^{-1}\left(\mathrm{~m}_{0}\right)$ to $\pi^{-1}\left(\mathrm{~m}_{1}\right)$. By smooth dependence of solutions of ordinary differential equations on initial conditions, it follows that this map is a diffeomorphism; it is called the parallel transport operator. 
Now let $\mathrm{m}(\mathrm{t}), \mathrm{t} \in[0,1]$ be closed path in $M$ satisfying $\mathrm{m}_{1}=\mathrm{m}_{0}$. The diffeomorphism of $\pi^{-1}\left(\mathrm{~m}_{0}\right)$ onto itself given by parallel transport along $\mathrm{m}(\mathrm{t})$ is called the holonomy of the path $\mathrm{m}(\mathrm{t})$. It is easy to see that parallel transport sends juxtaposition of loops based on $\mathrm{m}_{0}$ into the composition of diffeomorphisms of $\pi^{-1}\left(\mathrm{~m}_{0}\right)$. Thus the holonomy operation is a group homomorphism of $\mathcal{L}\left(\mathrm{m}_{0}\right)$, the loop group at $\mathrm{m}_{0}$, to the diffeomorphism group of the fiber $\pi^{-}$ ${ }^{1}\left(\mathrm{~m}_{0}\right)$; its image $\mathcal{H}\left(\mathrm{m}_{0}\right)$ is called the holonomy group at $\mathrm{m}_{0}$. It is straightforward to see that if $\mathrm{M}$ is connected, all holonomy groups are conjugate: $\mathcal{H}\left(\mathrm{m}_{0}\right)$ and $\mathcal{H}\left(\mathrm{m}_{1}\right)$ are conjugate by the parallel transport along any path connecting $\mathrm{m}_{0}$ to $\mathrm{m}_{1}$. Thus if $\mathrm{M}$ is connected, we speak of $\mathscr{H}$ , the holonomy group of the connection.

If $\pi: \mathrm{E} \rightarrow \mathrm{M}$ is a principal bundle with abelian structure group and $\Omega$ is the curvature of a principal connection $\Gamma$, the holonomy is given by the group element

$$
\text { holonomy }=\exp \left(-\iint \mathrm{d} \Gamma\right)=\exp \left(-\iint \Omega\right) \text {, }
$$

where the integral is taken over any two-dimensional submanifold in $M$ whose boundary is $m(t)$, assuming one exists.

\section{\$4 The Hannay-Berry Connection}

We summarize below the main properties and the construction procedure of the HannayBerry connection. The proofs will be omitted since they are straightforward generalizations of those in Montgomery [1988] once the following theorem is proved.

4.1 Theorem Averaging of Connections Let $\pi: \mathrm{E} \rightarrow \mathrm{M}$ be a fiber bundle and let $\Gamma$ $\in \Omega^{1}(\mathrm{E}, \mathrm{V})$ be an Ehresmann connection. Suppose a compact Lie group $\mathrm{G}$ acts on $\mathrm{E}$ by bundle transformations, not necessarily covering the identity. Then the average $\langle\Gamma\rangle$ of $\Gamma$ (see equation (3)) is also an Ehresmann connection. Moreover the G-action commutes with the action of parallel translation with respect to $\langle\Gamma\rangle$.

The proof is a consequence of the following two facts: 1 The average of a collection of points sitting inside a convex set is again an element of that set. 2 The set of connections is a convex (in fact affine) subset of the set of $(2,1)$ tensor field on E. (For example, these two facts are used when one averages a Riemannian metric to obtain a new metric for which $G$ acts by isometries.)

Let $\pi: \mathrm{E} \rightarrow \mathrm{M}$ be a Poisson fiber bundle, i.e., $\pi$ is a surjective submersion, all fibers are Poisson manifolds, and the transition functions are Poisson maps. Let $G$ be a Lie group. A family of Hamiltonian G-actions on $E$ is a smooth (left) G-action on $E$ such that each fiber $\pi^{-1}(\mathrm{~m})$ is invariant under the action and the action restricted to each fiber is Hamiltonian, i.e. it is Poisson and it admits a fiberwise momentum map $\mathbf{I}: \mathrm{E} \rightarrow \mathrm{g}^{*}$. This means that for each $\xi \in$ g, we have 


$$
\xi_{E}(p)=X_{T \xi}(p)
$$

where $I^{\xi}$ denotes the real valued function defined by $I^{\xi}(p)=\mathbf{I}(p) \cdot \xi$, for $p \in E$ and $\xi \in g$, $\xi_{\mathrm{E}}(\mathrm{p})=\left.\frac{\mathrm{d}}{\mathrm{d} \varepsilon}\right|_{\mathrm{t}=0}(\exp \varepsilon \xi \cdot \mathrm{p})$ is the infinitesimal generator of the action defined by $\xi$ and $\mathrm{X}_{\mathrm{I}}(\mathrm{p})$ is the Hamiltonian vector field on the fiber through $p$ defined by the function $I^{\xi}: E \rightarrow \mathbb{R}$. restricted to this fiber. Since the action on each fiber is Hamiltonian, the symplectic leaves of the fiber are G-invariant. Also, note that the Casimirs are G-invariant.

An Ehresmann connection on $\pi: \mathrm{E} \rightarrow \mathrm{M}$ is called Poisson if its horizontal lift hor $_{0}$ is a Poisson bracket derivation, i.e.

$$
\left(\text { hor }_{0} Z\right)[\{f, h\}]=\left\{\left(\text { hor }_{0} Z\right)[f], h\right\}+\left\{f,\left(\text { hor }_{0} Z\right)[h]\right\}
$$

for all $f, h: E \rightarrow \mathbb{R}$ and $Z \in \mathbb{X}(M)$. Equivalently, this says that

$$
\mathbf{D}_{0}\{f, h\} \cdot X=\left\{D_{0} f \cdot X, h\right\}+\left\{f, D_{0} h \cdot X\right\}
$$

for all $X \in \mathbb{X}(E)$, where $D_{0}$ is the covariant differentiation defined by hor ${ }_{0}$.

For example, let

$$
\operatorname{hor}_{0} \mathrm{Z}=\mathrm{X}_{-p \cdot \mathrm{Z}}+\mathrm{Z}
$$

be the Cartan connection on $\mathrm{E}=\mathrm{T}^{*} \mathrm{Q} \times \mathrm{M}$ defined in $\$ 2$. Then

$$
D_{0} f \cdot Z=\{f,-P \cdot Z\}
$$

(The bracket is defined by restricting $\mathrm{f}$ to $\mathrm{T}^{*} \mathrm{Q} \times\{\mathrm{m}\}$.) It follows from the Jacobi identity that the Cartan connection is Poisson.

4.2 Definition The Hannay-Berry (HB) connection induced by a Poisson-Ehresmann connection hor ${ }_{0}$ is the Ehresmann connection on $\pi: \mathrm{E} \rightarrow \mathrm{M}$ obtained by averaging hor . $^{\text {. (See }}$ Equation (3).) We will let $\mathbf{D}$ denote its covariant derivative, hor its horizontal lift, and $\gamma \in$ $\Omega^{1}(\mathrm{E}$, ker $\mathrm{T} \pi)$ its connection one-form.

If hor ${ }_{0}$ is the Cartan connection, then we will call the resulting average the CartanHannay-Berry (CHB) connection. Its horizontal lift was given in $\$ 2$ :

$$
\text { hor } \cdot \mathrm{Z}=\mathrm{X}_{-\langle P \cdot \mathrm{Z}\rangle}+\mathrm{Z} \text {. }
$$


If $\lambda$ is any tensor field defined along (as opposed to on) a G-invariant submanifold of $E$, its average $\langle\lambda\rangle$ is the smooth G-invariant tensor field of the same type defined along the same submanifold by

$$
\langle\lambda\rangle=\frac{1}{|G|} \int_{\mathrm{G}}\left(\Phi_{\mathrm{g}}^{*} \lambda\right) \mathrm{dg}, \text { where }
$$

$\Phi: \mathrm{G} \times \mathrm{E} \rightarrow \mathrm{E}$ is the $\mathrm{G}$-action on $\mathrm{E}$. Note that $\langle\lambda\rangle$ is a G-invariant tensor field.

The following two propositions summarize the important properties of the HB connection.

4.3 Proposition Suppose $\mathrm{G}$ is compact and connected. Then the $\mathrm{HB}$ connection satisfies the following properties:

i It is Poisson.

ii If $\mathrm{v} \in \mathrm{T}_{\mathrm{m}} \mathrm{M}$ then its $\mathrm{HB}$ horizontal lift is given by

$$
\text { hor } \mathrm{v}=\left\langle\operatorname{hor}_{0} \mathrm{v}\right\rangle \text {. }
$$

iii hor $\mathrm{Z}=$ hor $_{0} \mathrm{Z}+\mathrm{X}_{\mathrm{K} \cdot \mathrm{Z}}$, for a smooth function $\mathrm{K} \cdot \mathrm{Z}: \mathrm{E} \rightarrow \mathbb{R}$ and $\mathrm{Z} \in \mathbb{X}(\mathrm{M})$. iv The connection one-form of the $\mathrm{HB}$ connection is given by

$$
\Gamma(v)=\Gamma_{0}(v)-X_{K \cdot T \pi(v)}(p), \text { for } v \in T_{p} E
$$

$\mathbf{v}\langle\mathrm{K} \cdot \mathrm{Z}\rangle$ is a fiberwise Casimir function.

vi $\mathbf{D}\langle\lambda\rangle=\langle\mathbf{D} \lambda\rangle=\langle\mathbf{d} \lambda\rangle \circ \mathbf{P}_{\mathrm{hor}}$, for any $\lambda \in \Omega^{\mathrm{k}}(\mathrm{E}), \mathrm{k}=0,1, \ldots$, where $\mathbf{P}_{\mathrm{hor}}$ is the horizontal projection relative to hor.

vii $\mathrm{DI}^{\xi}$. hor $\mathrm{Z}=\left\langle\mathrm{D}_{0} \mathrm{I}^{\xi}\right.$. hor $\left.\mathrm{Z}\right\rangle$ is a Casimir for each $\xi \in \mathrm{g}, \mathrm{Z} \in \mathbb{X}(\mathrm{M})$. Moreover, $\mathbf{D I}=\left\langle\mathbf{D}_{0} \mathbf{I}\right\rangle$ and hence $\mathbf{D I}=0$ iff $\left\langle\mathbf{D}_{0} \mathbf{I}\right\rangle=0$.

Remark Property vi holds for any averaged connection. The rest of the properties are consequences of the following general principle:

If $\mathrm{E}$ has structure group $\mathcal{G}$, and both $\Gamma_{0}$ and $\mathrm{G}$ preserve this structure, then $\left\langle\Gamma_{0}\right\rangle$ also preserves this structure. In the $\mathrm{HB}$ case, $\mathcal{G}$ is the group of Hamiltonian automorphisms of the fiber.

4.4 Proposition Let hor be the horizontal lift of an Ehresmann connection on $\pi: \mathrm{E} \rightarrow \mathrm{M}$ satisfying

a $\mathbf{D I}=0$, where $\mathbf{D}$ is the covariant differentiation given by hor; this says that parallel translation relative to hor preserves the level sets of $\mathbf{I}$;

b hor $\mathrm{Z}=$ hor $_{0} \mathrm{Z}+\mathrm{X}_{\mathrm{K} \cdot \mathrm{Z}}$ for some smooth function $\mathrm{K} \cdot \mathrm{Z}: \mathrm{E} \rightarrow \mathbb{R}$, where $\mathrm{Z} \mapsto \mathrm{K} \cdot \mathrm{Z}$ is linear; 
c $\langle\mathrm{K} \cdot \mathrm{v}\rangle$ is a Casimir function on $\pi^{-1}(\mathrm{~m})$. where $\mathrm{v} \in \mathrm{T}_{\mathrm{m}} \mathrm{M}$; replacing $\mathrm{K} \cdot \mathrm{v}$ by $\mathrm{K} \cdot \mathrm{v}-\langle\mathrm{K} \cdot \mathrm{v}\rangle$, we can assume that this Casimir is zero.

Then i such a connection is unique;

ii such a connection exists if and only if the 'adiabatic condition'

$$
\left\langle\mathbf{D}_{0} \mathbf{I}\right\rangle=0
$$

holds, in which case the connection equals the Hannay-Berry connection.

Remarks 1 According to Proposition 4.3iii the covariant derivative of a function $f$ with respect to the $\mathrm{HB}$-connection is

$$
\text { Df } \cdot \mathrm{u}=\mathrm{D}_{0} \mathrm{f} \cdot \mathrm{u}+\{\mathrm{f}, \mathrm{K} \cdot \mathrm{u}\}
$$

2 We call condition (A) the 'adiabatic condition' because in the context of a family of completely integrable systems this equality is the content of the classical adiabatic theorem.

3 If $\mathrm{G}$ is semisimple and $\mathrm{I}$ is equivariant the 'adiabatic condition' (A) automatically holds. Consequently, the HB connection satisfies properties $\mathbf{a}, \mathbf{b}$, and $\mathbf{c}$ of Proposition 4.4.

Indeed, properties vi and vii of Proposition 4.3 we have $\mathrm{DI}=\langle\mathrm{DI}\rangle=\mathrm{D}\langle\mathbf{I}\rangle$. By equivariance $\langle\mathbf{I}\rangle^{\xi}=\mathbf{I}^{\langle\xi\rangle}$, for every $\xi \in \mathrm{g}$. Now $\langle\xi\rangle$ is an Ad-invariant vector. Since $\mathrm{G}$ is semi-simple, the adjoint representation is irreducible and so $\langle\xi\rangle=0$; consequently $\langle\mathbf{I}\rangle=0$, and thus $\mathbf{D I}=0$.

4 Suppose, for each $v \in T_{m} M$ there is a function $\tilde{\mathrm{K}} \cdot v: \pi^{-1}(\mathrm{~m}) \rightarrow \mathbb{R}$ satisfying

$$
\mathrm{dI}^{\xi} \cdot \operatorname{hor}_{0} \mathrm{v}+\left\{\mathrm{I}^{\xi}, \tilde{\mathrm{K}} \cdot \mathrm{v}\right\}=0
$$

for all $\xi \in \mathrm{g}$. Set $\mathrm{K} \cdot \mathrm{v}=\tilde{\mathrm{K}} \cdot \mathrm{v}-\langle\tilde{\mathrm{K}} \cdot \mathrm{v}\rangle$ and hor $\mathrm{v}=\operatorname{hor}_{0} \mathrm{v}+\mathrm{X}_{\mathrm{K} \cdot \mathrm{v}}$. Assume $\left\langle\mathbf{D}_{0} \mathbf{I}\right\rangle=0$, so hor defines the HB connection. This is proved by verifying the conditions in Proposition 4.4.

We now specialize to the case of the Cartan connection, ending with the main theorem of this section. Assume that a Lie group $\mathrm{G}$ acts on $\mathrm{T}^{*} \mathrm{Q}$ on the left with equivariant momentum map $\mathrm{I}: \mathrm{T}^{*} \mathrm{Q} \rightarrow \mathrm{g}^{*}$. Then $\mathrm{G}$ defines a family of Hamiltonian actions on $\mathrm{T}^{*} \mathrm{Q} \times \mathrm{M}$ by letting $\mathrm{G}$ act trivially on $M$. Its parametrized momentum map is simply $I$ thought of as a function of two variables, independent of $M$. Notice that the adiabatic condition

$$
\left\langle\mathbf{D}_{0} \mathbf{I}\right\rangle=0
$$

holds for the Cartan connection. This is a consequence of the following lemma, applied to the functions $\mathrm{f}=\boldsymbol{P} \cdot \mathrm{Z}$. 
4.5 Lemma Let $\pi: \mathrm{E} \rightarrow \mathrm{M}$ be a Poisson fiber bundle endowed with a family of Hamiltonian $\mathrm{G}$-actions with equivariant parametrized momentum map $\mathrm{I}: \mathrm{E} \rightarrow \mathrm{g}^{*}$. Then for any $\mathrm{f}: \mathrm{E} \rightarrow \mathbb{R}$ we have $\left\langle\left\{\mathbf{I}^{\xi}, \mathrm{f}\right\}\right\rangle=0$ for all $\xi \in \mathbf{g}$.

Proof Apply the Fubini theorem and use the fact that $\left\langle\frac{\partial \mathrm{f}}{\partial \theta}\right\rangle=0$ when $\mathrm{G}=\mathrm{S}^{1}$.

4.6 Main Theorem The $\mathrm{CHB}$ connection is a Poisson connection for $\mathrm{T}^{*} \mathrm{Q} \times \mathrm{M} \rightarrow \mathrm{M}$. Its horizontal lift is given by

and satisfies

$$
\text { hor } Z=\left(-X_{\langle P, Z\rangle}, Z\right)
$$

$$
\mathbf{D I}=0
$$

together with the rest of properties a, b, $\mathbf{c}$ of Proposition 4.4, and i - vii of Proposition 4.3. Its holonomy defines the Hannay angles of a slowly moving constrained mechanical system.

This follows from the adiabatic condition and Propositions 4.3 and 4.4.

Recall that the holonomy of a closed loop relative to an Ehresmann connection is the diffeomorphism of the fiber given by parallel translation. In the case of the Hannay-Berry connection induced by a Cartan connection, the fiber is $T^{*} \mathrm{Q}$. Thus if $\mathrm{c}(\mathrm{t})$ is a closed loop of embeddings of $\mathrm{Q}$ in $S$ the differential equations for the horizontal lift of $c(t)$ in $T^{*} Q$ are Hamilton's equations for the Hamiltonian $-\left\langle P \cdot \frac{\mathrm{dc}}{\mathrm{dt}}\right\rangle$.

\section{$\$ 5$ Concluding Remarks}

An expanded version of the present paper is available. In addition to the theory for moving systems described here, we also discuss the following.

\section{\$5 Symplectic Fiber Bundles}

Suppose the bundle $\mathrm{E} \rightarrow \mathrm{M}$ of $\$ 4$ is a symplectic fiber bundle, that is, its fibers are symplectic, so that each fiber has a symplectic form $\omega_{\mathrm{f}}$ and these fit together smoothly. It is a

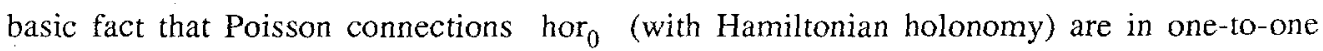
correspondence with closed extensions $\omega$ of the fiber 2 -form $\omega_{f}$ to all of $E$. The correspondence is

$$
\text { Hor }_{0}=\text { Vert }^{\omega}
$$


where ${ }^{\omega}$ denotes the $\omega$-orthogonal complement. (This fact is due to Gotay, et al. [1983] and was presented to us in this more digestible fashion by V. Guiliemin.)

This means that our averaged connection can be obtained by averaging the 2 -form:

$$
\text { Hor }=\operatorname{Vert}^{(\omega)} \text {. }
$$

This approach has many simplifying virtues, and is the one taken by Golin, et al. [1988]. For example, if $\omega=-\mathbf{d} \theta$, it immediately leads to

$$
I^{\xi}=\left\langle i_{\xi_{E}} \theta\right\rangle
$$

for the momentum map. One checks directly that this is the standard formula $\oint$ pdq for actions in the case where the group is abelian. Also, these automatically satisfy the adiabatic condition $\left\langle D_{0} I\right\rangle$ $=0$.

\section{\$B Normal Forms}

Suppose in $\$ 2$, that the external potential $U$ is independent of $M$, and that we are averaging over the flow of $\mathrm{H}_{0}$. Then

$$
\left[\mathrm{X}_{\mathrm{H}_{0}} \text {, hor } \mathrm{Z}_{\mathrm{t}}\right]=0 \text {, }
$$

and our approximate dynamics is defined by the vector field

$$
\left(\mathrm{X}_{\mathscr{H}}, \mathrm{Z}_{\mathrm{t}}\right)=\mathrm{X}_{\mathrm{H}_{0}}+\text { hor } \mathrm{Z}_{\mathrm{t}}
$$

It follows that in this case the $H B$ connection hor $Z_{\mathfrak{t}}$, can be viewed as the second term in a normal form for the true dynamics (2.4).

To see this, recall that the normal form for a (time-independent) vector field $X=X_{0}+\varepsilon X_{1}$ $+\mathrm{O}\left(\varepsilon^{2}\right), \varepsilon$ a formal parameter, is obtained by changing variables using the flow of $X_{0}$. In the new coordinates $X=X_{0}+\varepsilon Y_{1}$ where $\left[X_{0}, Y_{1}\right]=0$ and $Y_{1}$ is the average of $X_{1}$ with respect to the flow of $X_{0}$. See Churchill, Kummer, Rod [1983], or Cushman [1988] for more regarding this point of view on normal forms.

\section{\$5C Reconstruction of Reduced Motions}

We begin as in $\$ 2$ with a simple mechanical system $H=K+V$, together with a group $G$ acting in such a way as to preserve $\mathrm{K}$ and $\mathrm{V}$. Here $\mathrm{K}$ is kinetic energy on $\mathrm{T}^{*} \mathrm{Q}$. We have the momentum map $\mathbf{I}: \mathrm{T}^{*} \mathrm{Q} \rightarrow \mathrm{g}^{*}$, and the reduced spaces $\left(\mathrm{T}^{*} \mathrm{Q}\right)_{\mu}=\mathrm{I}^{-1}(\mu) / \mathrm{G}_{\mu}$ for $\mu \in \mathrm{g}^{*}$; see Marsden and Weinstein [1974]. 
Motions with momentum $\mu$ push down to solutions to the reduced Hamiltonian system on $\left(\mathrm{T}^{*} \mathrm{Q}\right)_{\mu}$. (One example of this is the planar Kepler problem. There $\mathbf{I}$ is angular momentum, and $\left(\mathrm{T}^{*} \mathrm{Q}\right)_{\mu}$ is coordinatized by the radius and the radial momentum variables.) The basic problem is to reconstruct the original dynamics from the reduced dynamics. Consider the bundle

$$
\mathbf{I}^{-1}(\mu) \rightarrow\left(T^{*} Q\right)_{\mu}
$$

with fiber $\mathrm{G}_{\mu}$ representing the "angles" we ignore in reducing. The idea is then to horizontally lift the solution curve on the reduced space, to a curve $\bar{c}(t)$ in $I^{-1}(\mu)$. Writing

$$
\mathrm{c}(\mathrm{t})=\mathrm{g}(\mathrm{t}) \cdot \overrightarrow{\mathrm{c}}(\mathrm{t})
$$

we get a first order differential equation for the desired angles $g(t) \in G_{\mu}$. If $G_{\mu}$ is abelian (as it must be generically) this equation is solvable in quadratures, and the reconstruction is complete.

The new ingredient needed is a connection with which to obtain the horizontal curve $\bar{c}$. We construct this connection out of the natural connection on $Q \rightarrow Q / G_{\mu}$. This is the connection for which

$$
\operatorname{Hor}_{q}=\left(T_{q} G_{\mu} \cdot q\right)^{\perp}
$$

where $\perp$ is taken relative to the kinetic energy metric. (For this to be an honest connection, we must assume that the $\mathrm{G}$ action is free.)

\section{\$5 Tower of Bundles}

The HB connection and the 'reconstruction connection' just described can be put together to define a connection on each post of the tower of bundles

$$
\mathbf{I}^{-1}(\mu) \rightarrow \mathbf{I}^{-1}(\mu) / G_{\mu} \rightarrow M
$$

The HB connection is a connection for $I^{-1}(\mu) \rightarrow M$, and the reconstruction connection gives a fiber-wise connection on

$$
\mathbf{I}^{-1}(\mu)_{m} \rightarrow \mathbf{I}^{-1}(\mu)_{m} / G_{\mu}
$$

for each $\mathrm{m} \in \mathrm{M}$. The two connections can be put together thus defining connections on either $\mathbf{I}^{-1}(\mu) \rightarrow \mathbf{I}^{-1}(\mu) / G_{\mu}$, or, on the bundle of sympelctic reduced spaces $\mathbf{I}^{-1}(\mu) / G_{\mu} \rightarrow M$. It is hoped that these connections can help give fairly complete descriptions of the dynamics in the averaging approximation. This situation appears to be appropriate, for example, for the dynamics 
of slowly moving coupled rigid bodies (e.g., coupled rigid bodies in orbit about the earth). The first bundle in $(T)$ is the bundie corresponding to internal versus overall rotational modes and the second to the parametrized motion of the system. See also $\$ \mathbf{5 E}$.

\section{\$5E An Optimal Control Problem}

Go back to the setting of $\$ 5 \mathrm{C}$ but now view it as the set-up for a control problem. The desired result will be a holonomy $\mathrm{g} \in \mathrm{G}$. The control parameter space will be $\mathrm{Q} / \mathrm{G}$, based at $\mathrm{q}_{0}$, whose holonomy is $\mathrm{g}$. The optimal control problem is to find the shortest such loop.

One of the motivating problems is the following. Let $Q$ be the configuration space for a sytstem of coupled planar rigid bodies relative to an inertial frame. $G=S \mathbf{O}(2)$ represents rigid rotations. Then $\mathrm{Q} / \mathrm{G}$ is the set of shapes, which can be parameterized by the hinge angles, which we assume that we can control.

The holonomy $\mathrm{g}$ resulting from a given shape change is exactly the rigid rotation which the system undergoes after a given manipulation of the hinge joints. the connection with respect to which we calculate the holonomy is the constraint: "angular momentum equals zero". This is the same connection as the one alluded to in $\$ 5 \mathrm{C}$, i.e. the connection defined by kinetic energy on $Q$.

The optimal control question has been answered by Montgomery [1989] in the sense that it has been reduced to an o.d.e. Somewhat surprisingly this is the same o.d.e. which governs the motion of a "charged particle in an electromagentic field" (on the space Q/G).

\section{References}

V. Arnold [1978] Mathematical Methods of Classical Mechanics, Graduate Texts in Mathematics, 60, Springer Verlag.

M. Berry [1984] Quantal phase factors accompanying adiabatic changes, Proc. Roy. Soc. London A 392, 45-57.

M. Berry [1985] Classical adiabatic angles and quantal adiabatic phase, J. Phys. A: Math. Gen. $18,15-27$.

M. Berry [1988] The geometric phase. Scientific American, December, 1988.

M. Berry and J. Hannay [1988] Classical non-adiabatic angles, J. Phys. A: Math. Gen 21, 325333.

E. Cartan [1923] Sur les varietes a connexion affine et theorie de relativite generalizée, Ann. Ecole Norm. Sup. 40, 325-412, 41, 1-25.

R. Churchill, M. Kummer, D. Rod [1983] On averaging, reduction, and symmetry in Hamiltonian system, J. Diff. Eq. 49, 359-414.

R. Cushman [1988] A survey of normalization techniques applied to perturbed Keplerian system, Dept. of Math., Univ. of Utrecht, preprint 496. 
S. Golin, A. Knauf, S. Marmi [1988] The Hannay angles: geometry, adiabaticity, and an example, preprint.

M. Gotay, R. Lashof, J. Sniatycki, A. Weinstein [1983] Closed forms on symplectic fiber bundles, Comm Math. Helv. 58, 617-621.

J. Hannay [1985] Angle variable holonomy in adiabatic excursion of an integrable Hamiltonian, $J$. Phys. A: Math. Gen 18, 221-230.

J.E. Marsden, and T. J.R. Hughes [1983] Mathematical Foundations of Elasticity, Prenctice Hall.

J.E. Marsden, R. Montgomery, T. Ratiu [1989] Reduction, Symmetry, and Berry's Phase in Mechanics, preprint.

J.E. Marsden and A. Weinstein [1974] Reduction of symplectic manifolds with symmetry. Rep. Math. Phys. 5, 121-130

R. Montgomery [1988] The connection whose holonomy is the classical adiabatic angles of Hannay and Berry and its generalization to the non-integrable case, Comm. Math. Phys. 120, 269-294

R. Montgomery [1989] Shortest loops with a fixed holonomy, MSRI Berkeley, preprint.

Jerrold E. Marsden

Department of Mathematics

University of California

Berkeley, CA 94720

Richard Montgomery

MSRI

1000 Centennial Drive

Berkeley, CA 94720

Tudor Ratiu

Department of Mathematics

University of California

and MSRI

Santa Cruz, CA 94305

1000 Centennial Drive

Berkeley, CA 94720 